\title{
Validity of spirometry performed online
}

\author{
J.F. Masa*,\#, M.T. González*, R. Pereira*\#, M. Motađ, J.A. Riesco*, J. Corral*,", \\ J. Zamorano ${ }^{+}$, M. Rubio ${ }^{\#, \S}$, J. Teran ${ }^{\#, f}$ and R. Farré ${ }^{\#, * *}$
}

ABSTRACT: Spirometry is essential for the diagnosis and management of common respiratory diseases. However, its use and quality are low in primary care. An important reason for this is the technical difficulty in performing conventional spirometry. If high-quality spirometry could be performed online, from the pulmonary function laboratory in hospitals, most of the technical problems could be solved. The aim of the present study was to compare spirometries performed online by remote technicians with conventional spirometry.

This was a controlled, randomised crossover study of 261 patients referred from primary care centres for pulmonary consultation. They were randomised to undergo either conventional or online spirometry. The technician, located remotely, controlled the spirometer computer. Using a teleconference link, the technician guided the patient through the spirometry. The comparison between conventional and online spirometries was performed on intention to treat and per protocol bases for spirometric values and quality criteria. Agreement between the two spirometric methods was assessed with a Bland-Altman plot. A subpopulation of off-range patients was also characterised. Finally, intra- and interobserver agreement was evaluated using the intraclass correlation coefficient.

No clinically significant differences were seen between the online and conventional spirometric values in both the intention to treat and per protocol analyses. The agreement in Bland-Altman analysis was poorer for intention to treat than for the per protocol analysis. The latter had a lower percentage of off-range patients and high agreement to determine abnormal spirometry in the offrange group. Conventional spirometry had a higher percentage of patients with spirometric quality criteria although the quality criteria difference was only $5.9 \%$, when both procedures were the first to start. Very good agreement was found between intra- and interobserver reliability.

Spirometry performed online from a hospital can be an adequate alternative to conventional spirometry for primary care centres.

KEYWORDS: Chronic obstructive pulmonary disease, spirometry, telemedicine, telespirometry

orced spirometry is a basic tool to explore the function of the respiratory system, confirm normality, detect and classify potential respiratory patterns (restrictive or obstructive), and indicate the degree of severity of the disease. Specifically, spirometry is essential to the diagnosis and management of very common diseases, such as asthma and chronic obstructive pulmonary disease (COPD) [1]. COPD is currently the fourth most common cause of death in the developed world and it could become the third [2]. Early diagnosis of COPD could change the natural evolution of the disease $[3,4]$. However, a considerable number of COPD patients remain underdiagnosed $[1,5]$.

In this context, the performance of spirometry in primary care has been encouraged for improving the diagnosis and characterisation of COPD and asthma patients [6-10]. However, this approach has limitations, because the routine use of spirometry in primary care is infrequent [11] and technical quality is poor [12-14], according to several reports of quality assessment [15]. This fact is largely explained by the difficulty encountered by primary care staff in performing technically acceptable spirometries [12]. Accordingly, various approaches have been proposed to extend the routine application of spirometry in primary care.

One of the approaches consists of using handheld office spirometers, since they are portable, less expensive and easier devices to use $[16,17]$. However, some studies have found that using these devices could result in a technically inferior spirometry as compared to reference spirometers in the hospital pulmonary function laboratory $[18,19]$. Consequently, these portable devices may be acceptable for COPD screening [20], but relevant concerns remain about their use as an alternative to high-quality spirometers [21].

\section{AFFILIATIONS}

*Pulmonary Service,

+Research Unit, San Pedro de Alcántara Hospital,

SPrimary Care, Center Zone, Cáceres, \#CIBER de Enfermedades

Respiratorias (CIBERES), Instituto de Salud Carlos III, Madrid,

"Mathematics Dept, Extremadura University, Badajoz,

${ }^{f}$ Pulmonary Service, General Yagüe

Hospital, Burgos, and

**Biophysics and Bioengineering Unit, Medicine Faculty, BarcelonaIDIBAPS University, Barcelona, Spain.

CORRESPONDENCE

J.F. Masa

C/Rafael Alberti 12

10005 Cáceres

Spain

E-mail: fmasa@separ.es

Received:

Jan 222010

Accepted after revision:

July 052010

First published online:

July 222010 
Given that the most common spirometric failure in general practice is found at the end-of-test point, that is, when calculating forced vital capacity (FVC) and its ratio with forced expiratory volume in $1 \mathrm{~s}$ (FEV1) (FEV1/FVC) [22], another approach consists of replacing the FVC with forced expiratory volume in $6 \mathrm{~s}$ (FEV6) [16]. However, discordance in the utility of FEV1/FEV6 compared with FEV1/FVC ratios has been reported [23], although a recent meta-analysis reached the conclusion that FEV1/FEV6 is a sensitive and specific alternative to FEV1/FVC for the diagnosis of airway obstruction [24].

Yet another approach for improving the technical quality of spirometry in primary care is enhancing staff training $[12,25,26]$. However, the results achieved, in terms of standard criteria, are not always satisfactory [12]. Moreover, a difficulty with this approach is that primary care staff training must be refreshed periodically $[12,25,26]$, with consequent logistic complications and economic costs, given the large number of professionals involved [21].

Whereas, to date, there is still an open debate about screening and high-quality diagnosis in primary care spirometry, new information and communication technologies can add new perspectives. Indeed, some studies have shown that it is possible for experienced patients to perform spirometry at home, with data transmitted telematically, to monitor asthma and the state of lung transplant recipients [27, 28]. If highquality spirometry could be performed online, with the technician in the pulmonary function laboratory in the reference hospital, and the patient and high-quality spirometer in the primary care centre, most of the technical and training issues could be solved, making it possible to perform highquality spirometries in naïve patients. Moreover, hospitals with well-developed pulmonary function laboratories could carry out spirometries at small hospitals around the world that do not have pulmonary function laboratories (e.g. Boston, MA, USA to Hong Kong, China).

The objective of the present study was to compare spirometry performed online, using remote technicians, with conventional spirometry.

\section{METHODS \\ Subjects}

Patients referred from primary care centres to pulmonary specialists in a tertiary hospital (San Pedro de Alcántara Hospital, Cáceres, Spain) were the target population. Other inclusion criteria were age 14-74 yrs and not previously having had a spirometry. Five patients were randomly chosen from those available per day. They were randomised to undergo one of two procedures: conventional or online spirometry. The exclusion criteria were psychophysical incapacity to carry out spirometry or refusal to sign a written consent form.

\section{Protocol}

This was a controlled, randomised, crossover study (conventional or online spirometry). Before the spirometry, each patient received written and graphical information about the procedure.

Spirometries were performed following standard recommendations and quality criteria (acceptability and repeatability) from the American Thoracic Society/European Respiratory
Society guidelines [15]. Accordingly, we used a spirometer (VMax 20; Viasys, San Diego, CA, USA) with the required flow and volume accuracy. The types and sequence of calibration checks were as follows: 1) volume, leak, volume and flow linearity (four different levels) daily; and 2) biological controls monthly. Both flow-volume and volume-time were displayed during the manoeuvre. The software automatically analysed the end-of-test criteria and reported when it was achieved during the manoeuvre. If the end of test criteria was not fulfilled, an alert message was shown before accepting the manoeuvre. If the $>6 \mathrm{~s}$ criterion was met, we did not exclude manoeuvres when subjects could not or would not continue further exhalation [15]. Other alert messages were regarding $>5 \%$ of extrapolated volume and repeatability (peak flow $>10 \%$, and FVC and FEV1 $>150 \mathrm{~mL}$ ).

Two technicians with similar experience (15 yrs) carried out the spirometries. $20 \mathrm{~min}$ after finishing the first spirometry (conventional or online), the second was performed.

\section{Online procedure}

The technician, located in a different and noncontiguous room from the patient, remotely controlled the computer with the spirometer software (Vmax SensorMedic; Viasys) by means of another computer and software (VNC Free Edition; RealVNC, Cambridge, UK). Two additional computers (one in each room) were used to perform a teleconference using common tools: a webcam (Logitech Quickcam USB; Logitech Barcelona, Spain) and software (Microsoft Netmeeting; Microsoft Corporation; Redmond, USA). We used an Ethernet network communication whose bandwidth was limited to full-duplex $512 \mathrm{Kbps}$ (NetLimiter; Locktime Software s.r.o.; Chisinau; Moldavia). Once the patient was invited to enter the spirometer room, they were alone except for telematic assistance. By teleconference, the technician asked the patient to sit on a chair in front of the computer, place the bacterial filter properly, hold the spirometer handpiece and initiate the spirometry.

\section{Outcomes}

The main outcomes were the comparison of numerical spirometric variables (FVC, FEV1 and FEV1/FVC ratio) and the percentage of patients with standard spirometric quality criteria [15] between conventional and online spirometries. Secondary outcomes were the comparison of the number of spirometric manoeuvres and the time spent on both spirometric procedures.

\section{Intra- and interobserver agreement}

One of the five patients studied per day was randomly selected to repeat the protocol, and there was a separate randomisation to determine if the same technician should repeat the process (intra-observer agreement) or the other technician should perform the second test (interobserver agreement). The initial procedure order (conventional or online) was maintained in every repetition.

\section{Statistical analysis}

The comparison between conventional and online spirometries was performed in two ways: intention to treat and per protocol. The intention to treat analysis included all randomised patients (whether or not spirometric quality criteria were achieved) and per protocol analysis included only patients fulfilling spirometric quality criteria (acceptability and repeatability) [15] in 


\section{TABLE 1 Characteristics of 261 patients studied}

\begin{tabular}{|c|c|}
\hline Measurement & Value \\
\hline Age yrs & $48.7 \pm 14.1$ \\
\hline \multicolumn{2}{|l|}{ Age group $\%$} \\
\hline $15-30$ yrs & 10.7 \\
\hline $31-45$ yrs & 32.2 \\
\hline $46-60$ yrs & 10.7 \\
\hline $61-75 \mathrm{yrs}$ & 26.1 \\
\hline Females \% & 39.8 \\
\hline BMI $\mathbf{k g} \cdot \mathrm{m}^{-2}$ & $28.7 \pm 6.1$ \\
\hline \multicolumn{2}{|l|}{ Consultation motive \% } \\
\hline Suspected asthma & 23.4 \\
\hline Suspected COPD & 13.8 \\
\hline Suspected sleep apnoea & 33.0 \\
\hline Dyspnoea/rule out illness & 20.3 \\
\hline Others & 9.6 \\
\hline \multicolumn{2}{|l|}{ Tobacco use \% } \\
\hline Smokers & 45.2 \\
\hline Ex-smokers & 18.0 \\
\hline Non-smokers & 36.8 \\
\hline Smoking expsoure cigarettes $\cdot$ day $^{-1}$ & $29.6 \pm 22.9$ \\
\hline Chronic cough \% & 20.3 \\
\hline \multicolumn{2}{|l|}{ MRC dyspnoea scale \% } \\
\hline 0 & 45.2 \\
\hline 1 & 39.5 \\
\hline$\|$ & 12.3 \\
\hline III & 3.1 \\
\hline $\mathrm{Sa}_{\mathrm{a}, \mathrm{O}_{2}} \%$ & $96.6 \pm 1.4$ \\
\hline \multicolumn{2}{|l|}{ Conventional spirometry, } \\
\hline FVC \% pred & $100.8 \pm 17.7$ \\
\hline FEV $1 \%$ pred & $94.3 \pm 20.1$ \\
\hline $\mathrm{FEV}_{1} / \mathrm{FVC}$ & $76.9 \pm 9.9$ \\
\hline Obstructive pattern \% & 16.6 \\
\hline Nonspecific pattern ${ }^{+} \%$ & 5.9 \\
\hline Starting online \% & 52.7 \\
\hline
\end{tabular}

Data are presented as mean $\pm \mathrm{SD}$, unless otherwise stated. BMI: body mass index; COPD: chronic obstructive pulmonary disease; MRC: Medical Research Council; $\mathrm{Sa}_{2} \mathrm{O}_{2}$ : arterial oxygen saturation; FVC: forced vital capacity; \% pred: $\%$ predicted; FEV 1 : forced expiratory volume in $1 \mathrm{~s} . ~ \#$ : from 259 patients. $\because$ : percentages according to European Respiratory Society predicted values [25]. ${ }^{+}$: FVC values below the lower limit of normal (LLN) plus normal FEV1/FVC ( $\left.F E V_{1} / F V C>L L N\right)$.

both conventional and online procedure groups. If no numerical value was obtained in the intention to treat group, we did not include these cases in the numerical analysis. However, we included these patients in the comparisons between online and conventional spirometric quality criteria. The statistical analysis was blinded, as data from online and conventional spirometries were labelled with a number. The meaning of these numbers was revealed in order to write this article.

We first compared the spirometric values and the secondary outcomes using the paired t-test (or Wilcoxon nonparametric test for non-normally distributed data) between conventional and online methods. Then, the mean difference between online and conventional spirometric values and secondary outcomes was correlated with age using a Spearman test to evaluate potential associations. In addition, we assessed agreement in spirometric values by Bland-Altman analysis. Patients with a difference in FVC and FEV1 greater or less than $150 \mathrm{~mL}$ (repeatability criteria) between online and conventional spirometries were identified as off-range. This group was compared with a nonoff-range group, for the variables shown in table 1 , to determine potentially discriminative variables (t-test or equivalent nonparametric test for continuous and Chi-squared for categorical variables). In the off-range group, the agreement for the determination of abnormal spirometry between conventional and online procedures was measured using the $\kappa$-test. Abnormal spirometry was defined by: 1) an obstructive (or mixed) pattern, defined as FEV1/FVC below the 5th percentile of the predicted value (lower limit of normal (LLN)) $[29,30]$; or 2) as a restrictive pattern requires total lung capacity measurement, FVC $<$ LLN and normal FEV1/FVC (FEV1/FVC $>$ LLN), as a nonspecific pattern [31].

The comparison of the percentage of patients with spirometric quality criteria between online and conventional spirometries was analysed using the MacNemar test in the total sample and when the sample was divided into the groups starting with online and conventional spirometry. In addition, Fisher's exact test was used to measure the percentage change (difference) between both spirometric methods in the two starting groups (online versus conventional).

Finally, intra- and interobserver agreement for repeated measurements of spirometric values was assessed by intraclass correlation coefficient (ICC) analysis.

\section{RESULTS}

283 patients were initially selected. $22(7.8 \%)$ patients were excluded due to lack of written consent and psychophysical incapacity (two patients were unable to understand the instructions and one patient had thoracic pain). The remaining 261 were randomised. In two patients (one in the conventional and the other in the online starting group), it was impossible to obtain any numerical spirometric values, because of nausea in one case and absence of cooperation in the other. In addition, a patient starting in the online group could not introduce the filter into the spirometer handpiece, so the online spirometry could not be performed. These three patients were analysed as nonacceptable spirometries (two in the online group and one in the conventional group) in the intention to treat analysis.

Excluded patients were similar to included subjects with regards to sex, body mass index, cigarettes smoked per day, arterial oxygen saturation $\left(\mathrm{Sa}_{\mathrm{a}} \mathrm{O}_{2}\right)$ and reason for consultation. They were statistically different in age (mean \pm SD $59 \pm 17$ yrs in excluded versus $49 \pm 14$ yrs in enrolled; $\mathrm{p}=0.01$ ).

Table 1 shows patient characteristics. Subjects were middleaged, predominantly male and overweight. Most of patients were smokers or ex-smokers with some degree of dyspnoea (generally mild). Obstructive patterns (based in conventional spirometry) were present in $16.6 \%$ of the sample and nonspecific pattern in $5.9 \%$, according to predicted values [32].

No significant differences were found between online and conventional spirometric values in either the intention to treat or per protocol analyses, with narrow $95 \%$ confidence intervals 
of the mean difference (table 2). Figure 1 shows the agreement of FVC and FEV1 between online and conventional methods using the Bland-Altman test. Although the biases between the two methods were small, the limits of agreement were higher in the intention to treat analysis (see Discussion for details). The difference between online and conventional spirometry was independent of measurement size.

In the intention to treat group, there were 54 (21\%) patients offrange in FVC and 40 (15\%) off-range in FEV1. In the per protocol group, there were 29 (14\%) patients off-range in FVC and $21(10 \%)$ off-range in FEV1. We did not find statistical differences in the comparisons between off-range and nonoffrange groups in the variables from table 1 in either the intention to treat or per protocol groups of patients. In the off-range group, the agreement for the determination of nonspecific and obstructive patterns between conventional and online spirometries was as follows: 1) in the intention to treat group, the $\kappa$-test results were $64 \%(p<0.001)$ for the nonspecific pattern and $77 \%(p<0.001)$ for the obstructive pattern; and 2) in the per protocol group, the $\kappa$-test results for the nonspecific pattern were $79 \%(\mathrm{p}<0.001)$ and for obstructive pattern was $86 \%(\mathrm{p}<0.001)$.

Both acceptability (conventional $88.9 \%$ and online $82 \%$; $\mathrm{p}=0.001$ ) and acceptability/repeatability (conventional $87.7 \%$ and on-line $81.6 \% ; \mathrm{p}=0.005)$ comparisons were statistically different $(\sim 6 \%$ better in the conventional procedure). The number of spirometric manoeuvres needed to achieve acceptability and repeatability criteria was marginally higher in the online method and this type of spirometry procedure tended to take longer time (table 2). No association was found between age and the differences in spirometric values, number of manoeuvres and time spent between the two spirometry methods.

The starting procedure had a weak influence on the percentage of patients with spirometric quality criteria (acceptability and repeatability). There were statistically significant differences between the two procedures in the online but not the conventional starting groups, showing a learning effect (table 3). However, the comparison between the two starting groups in the percentage change (difference) between the two spirometric methods was statistically significant in acceptability but did not achieve significance in acceptability and repeatability, indicating a limited learning effect. In addition, the difference between conventional and online when both procedures were the first to start was only $5.9 \%(85.5 \%$ for conventional and $79.6 \%$ for online) (table 3 ).

\section{Intra- and interobserver agreement}

Reliability for repeated measurements (ICC) is shown in table 4. High agreement was found in intra- and interobserver reliability.

\section{DISCUSSION}

This large, controlled, randomised study is the first published study to ascertain the validity of high-quality spirometry performed online. The spirometric values achieved online were very similar to those obtained using the conventional procedure. The reference (conventional) had a higher percentage of patients with spirometric quality criteria and a lower number of manoeuvres to achieve them.

From a practical point of view, the importance of the percentage of patients with spirometric quality criteria (acceptability and repeatability) is shown by the difference between conventional and online results when both procedures were the first to start, given that this situation reproduces normal conditions, that is, $85.5 \%$ for conventional and $79.6 \%$ for online, similar to the values obtained when analysing the total sample. In addition, there was a slight learning effect. In summary, both result in better quality criteria in the conventional spirometry. However, a difference of $5.9 \%$ is not crucial because variation in quality criteria between different respiratory function laboratories, or even between different technicians in conventional spirometry, exceeds this percentage [25, 33-35]. Conversely, although a greater number of spirometric manoeuvres and more time were needed for the online procedure in comparison with the conventional

TABLE 2 Spirometric values, number of manoeuvres and duration in conventional and online procedures

\begin{tabular}{|c|c|c|c|c|}
\hline Measurement & Online & Conventional & $\begin{array}{l}\text { Mean difference } \\
\qquad(95 \% \mathrm{Cl})\end{array}$ & $\begin{array}{c}\text { Correlation } \\
\text { coefficient }^{\#} \text { ( } p \text {-value) }\end{array}$ \\
\hline \multicolumn{5}{|l|}{ Intention to treat } \\
\hline FVC mL & $3695.2 \pm 1050.5$ & $3696.8 \pm 1047.6$ & $-1.6(-23.5-20.3)$ & $0.043(0.492)$ \\
\hline FEV1 mL & $2882.9 \pm 945.2$ & $2878.5 \pm 941.2$ & $4.5(-13.5-22.4)$ & $0.078(0.211)$ \\
\hline $\mathrm{FEV}_{1} / \mathrm{FVC} \%$ & $77.1 \pm 10.0$ & $76.9 \pm 9.9$ & $0.2(-0.2-0.6)$ & $0.046(0.466)$ \\
\hline \multicolumn{5}{|l|}{ Protocol $^{+}$} \\
\hline FVC mL & $3745.8 \pm 1077.3$ & $3734.7 \pm 1014.6$ & $11.2(-2.3-24.7)$ & $0.032(0.649)$ \\
\hline FEV1 mL & $2886.9 \pm 933.3$ & $2878.6 \pm 934.4$ & $8.2(-4.4-20.8)$ & $0.058(0.406)$ \\
\hline $\mathrm{FEV}_{1} / \mathrm{FVC} \%$ & $76.4 \pm 10.3$ & $76.4 \pm 10.4$ & $0.01(-0.3-0.3)$ & $0.025(0.718)$ \\
\hline Manoeuvres n & $5.0 \pm 1.7$ & $4.4 \pm 1.6^{\star \star \star}$ & $0.6(0.3-0.8)$ & $-0.114(0.104)$ \\
\hline Spirometric procedure duration min & $7.4 \pm 3.8$ & $6.7 \pm 3.6$ & $0.7(-1.4-1.6)$ & $-0.084(0.249)$ \\
\hline
\end{tabular}

Data are presented as mean $\pm \mathrm{SD}$, unless otherwise stated. FVC: forced vital capacity; FEV1: forced expiratory volume in $1 \mathrm{~s}$. ${ }^{*}$ : between mean difference and age; $\because: n=258 ;{ }^{+}: n=206 .{ }^{* * *}: p<0.001$. 

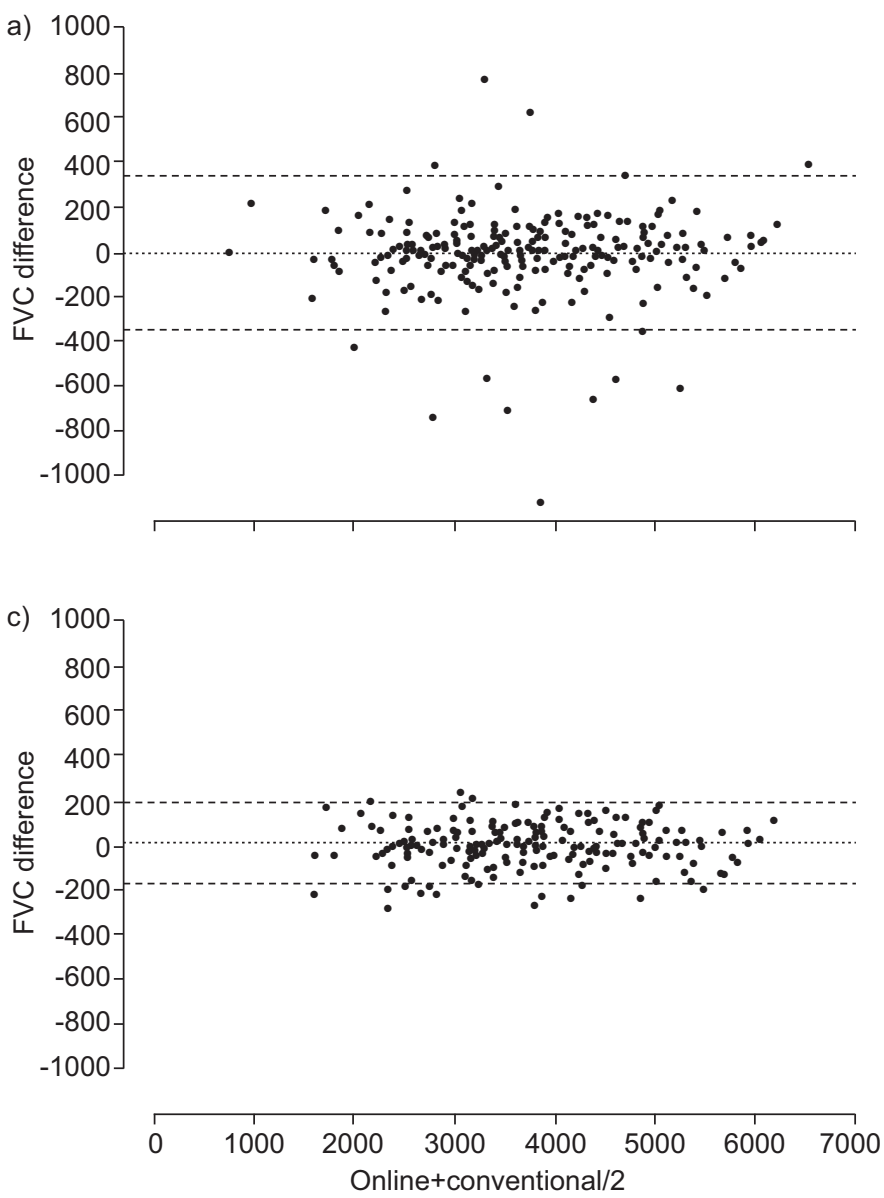
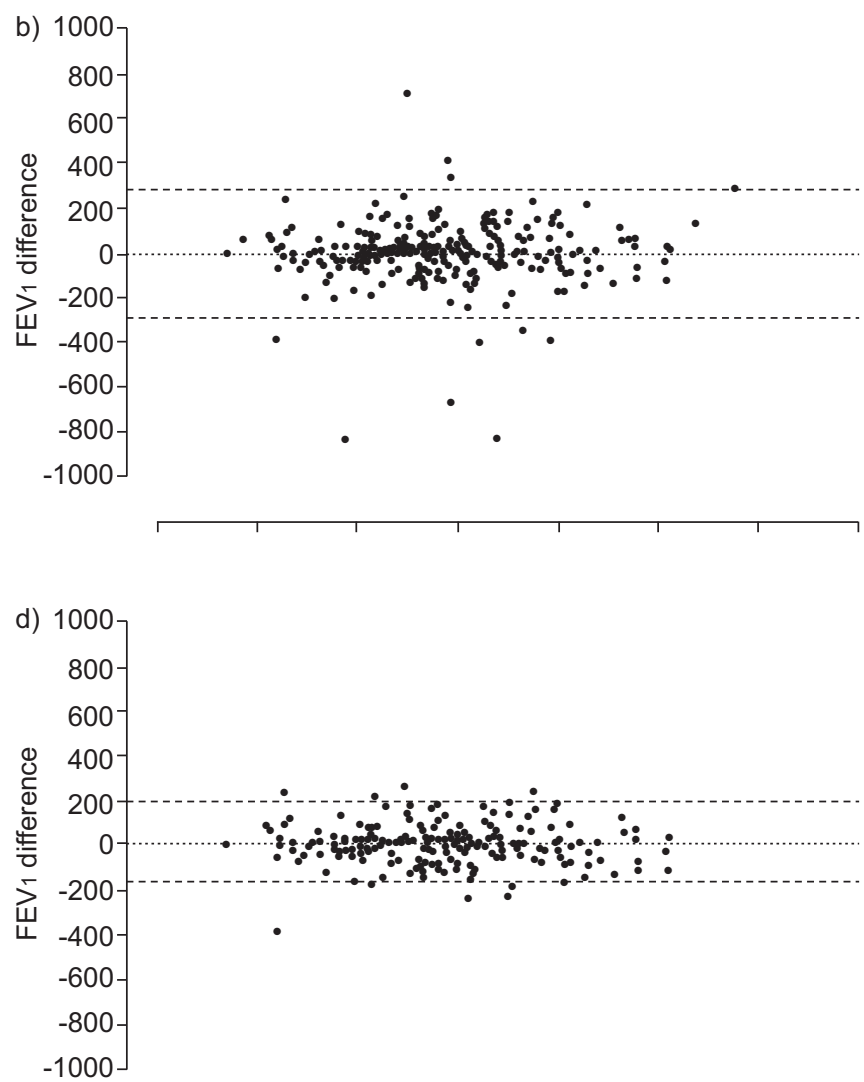

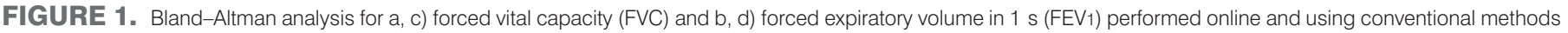
in the $\mathrm{a}, \mathrm{b}$ ) intention to treat and $\mathrm{c}, \mathrm{d}$ ) per protocol analyses. ...... mean difference; ---: limits of agreement.

procedure, the mean differences were 0.5 additional manoeuvres and $0.7 \mathrm{~min}$, which may be acceptable as well.

The spirometry is intrinsically variable between different tests in the same time period and patient: values $\leqslant 150 \mathrm{~mL}$ in both FVC and FEV1 are not considered significant [14]. When we calculate the agreement limits of the differences between the two methods in the Bland-Altman plot, we include this variability in addition to the variability between both types of spirometries. Since intrinsic variability is not systematic (it can occur in both methods of spirometry and vary among patients), the mean difference between conventional and online spirometries may be close to zero. For this reason, agreement limits can be overestimated. Based on the $95 \%$ confidence interval of the mean difference between methods, the probability of finding wide differences is low (table 2). In any case, agreement limits were higher in the intention to treat analysis. From a practical point of view, the most important analysis is per protocol, because spirometries without quality criteria are not taken into account.

In order to better characterise the discrepancies between the two spirometric methods, we identified off-range patients as having differences in FVC and FEV1 greater or less than $150 \mathrm{~mL}$ (repeatability criteria) between online and conventional spirometries. Although we did not find predictive variables for this off-range group, the frequency of off-range scores was low in the per protocol group (14\% in FVC and 10\% in FEV1). This frequency was very similar in the 54 patients in whom conventional spirometry was repeated twice for interand intra-observed agreement (12\% in FVC and $9 \%$ in FEV1), meaning that most of the numerical differences between online and conventional spirometry can be explained by the spirometric technique itself. In addition, high agreement between online and conventional spirometries was found to determine nonspecific and obstructive patterns in the off-range group.

New information and communication technologies can change many procedures that are carried out in daily clinical practice. Previous studies have shown that experienced patients can carry out spirometry at home to monitor FEV1 in asthma and the respiratory state of lung transplant recipients, transmitting data by telemetry $[27,28]$. Moreover, telemetry supervision of spirometry quality by specialists could be a teaching method for improving the quality of spirometry in primary care [13, 36]. The main subject of our study is the potential to relocate conventional spirometry to any place where a high-quality spirometer could be used efficiently, but its precise role in the primary care landscape has yet to be defined.

Proper technical training for performing spirometry is essential to achieving adequate quality. While the recommended 


\begin{tabular}{|c|c|c|c|c|c|c|c|}
\hline Acceptability \% & 79.6 & 90.5 & 0.000 & 84.7 & 87.1 & 0.581 & 0.027 \\
\hline Acceptability and repeatability \% & 79.6 & 89.8 & 0.001 & 83.9 & 85.5 & 0.791 & 0.058 \\
\hline
\end{tabular}

spirometry training time for pulmonary function laboratory technicians has been established at 6 months [37], there is no recommendation for primary care staff. The time employed in different studies is shorter and highly variable [12, 25, 26]. In addition, to provide adequate initial training and periodic inservice training for a large staff (e.g. all primary care centres) is expensive and logistically complex. As we understand it, the best option for large primary care centres, with many patients needing spirometric studies, may be to perform the spirometries in a place with proper and periodic training, perhaps by telematic learning $[8,13,36,38]$. However, in smaller primary care centres with fewer patients and resources to professionally train workers to carry out spirometry, the online alternative may be preferable. Similar reasoning could be used for small hospitals without pulmonary function laboratories.

Our randomised sample resulted in self-selection for relatively young patients. The main reason was the lack of written consent. The increased time needed to undergo an unknown test may be an important inconvenience for older patients. Nevertheless, this bias does not seem to influence our results, since age was not associated with the difference between both spirometric procedures in primary and secondary outcomes. On the other hand, most studied patients did not have altered spirometries because we selected the patients in order to obtain a representative sample of spirometrically naïve patients from the population referred by general practitioners for pulmonary consultation. In any case, the subgroup of patients with altered

\begin{tabular}{|c|c|c|c|}
\hline \multirow[t]{2}{*}{ TABLE 4} & \multicolumn{3}{|c|}{$\begin{array}{l}\text { Reliability for repeated measurements of } \\
\text { technicians }\end{array}$} \\
\hline & FVC & FEV $_{1}$ & FEV $1 /$ FVC \\
\hline \multicolumn{4}{|l|}{ Technician $1^{\#}$} \\
\hline Online & 0.977 & 0.988 & 0.983 \\
\hline Conventional & 0.994 & 0.958 & 0.973 \\
\hline \multicolumn{4}{|l|}{ Technician $2^{\circ}$} \\
\hline Online & 0.992 & 0.994 & 0.995 \\
\hline Conventional & 0.995 & 0.995 & 0.993 \\
\hline \multicolumn{4}{|c|}{ Technicians 1 versus $2^{+}$} \\
\hline Online & 0.997 & 0.997 & 0.956 \\
\hline Conventional & 0.998 & 0.993 & 0.977 \\
\hline
\end{tabular}

Data are presented as intraclass correlation coefficients. FVC: forced vital capacity; FEV1: forced expiratory volume in $1 \mathrm{~s} .{ }^{\#}: n=17 ;{ }^{\imath}: n=14 ;{ }^{+}: n=23$. spirometries showed no statistical differences between numerical variables (FVC, FEV1 and FEV1/FVC) registered with either spirometric method. Thus, the results should be similar in a sample with more severe spirometric alteration.

We included patients with suspected sleep apnoea to prevent the selection of patients referred from primary care for pulmonary consultation and because a spirometry at a hospital or primary care centre is now normally indicated in the diagnosis of these patients. In this group, $60 \%$ were smokers or ex-smokers, $39 \%$ had some grade of dyspnoea and $17 \%$ had chronic cough. Therefore, many of these patients could be referred for spirometric testing for reasons other than suspected sleep apnoea.

Online spirometry should be used via internet or intranet. Both, but particularly the internet, have important variability in the bandwidth available at any given point in time. Our required bandwidth was very low: $512 \mathrm{Kbps}$ of symmetric bandwidth (for upload and download traffic). It is easy to achieve a guaranteed minimum symmetric bandwidth of $512 \mathrm{Kbps}$ from internet or intranet providers over current infrastructure.

As already mentioned, once the patient entered the spirometer room, they had no technician present to help with proper placement of the bacterial filter, for holding the spirometer hand piece or for starting the process. Despite this apparent difficulty, only one patient was unable to put the bacterial filter in place. We believe that improving the written and graphical explanation provided before the test could improve patient cooperation and adjustment performance.

Light-headedness or dizziness are frequent during a spirometry procedure and these occurred during the telematic approach. All subjects were assisted telematically by technicians. Although during the study (624 complete spirometric procedures in 258 patients) we did not observe other important adverse problems, such as pneumothorax, bronchospasm or fainting, they obviously can occur. The latter has a special relevance because subjects could fall down and injure themselves. In our study, the technician was positioned close to the patients and they could respond rapidly, but in real practice the technician should be located far from the subject. For this reason, we understand that online spirometry requires some face-to-face assistance by a trained person in the spirometry room (see later).

We tried to replicate the conditions found outside the pulmonary function laboratory, but the simulation was not complete. Therefore, the following issues must be taken into 
account for the online spirometry to be judged practical. 1) Patients need attention before coming to the spirometer room in order to understand where and when the spirometry should be done, and must receive written information; an assistant and/or audio/video support should be available. 2) Management of the spirometer and its accessories and daily calibration check require trained staff. 3) Assistance for fainting and other potential adverse problems requires trained staff. 4) The application of an inhaled bronchodilator for assessing bronchodilation also requires trained staff. In summary, online spirometry cannot be done without face-toface support, but the level of training and staff qualification can be much lower for online than for conventional spirometry. In any case, adequate quality control procedures [39] should be implemented for online spirometry.

A limitation of our study is that the economic cost was not analysed. Nevertheless, it can be inferred from the additional equipment required: a computer with a basic webcam for teleconferencing in the spirometer room, two computers (one with a basic webcam) for remote control of spirometer software and teleconferencing in the technician's room (respiratory function lab) and an intra- or internet connection. Regarding staff costs, the previously mentioned resources spent on direct, face-to-face support by the hospital technician will be saved. Due to the fact that the training and salary of a hospital technician should be higher than that of primary care staff providing face-to-face assistance, additional primary care staff should not result in additional costs, at least in theory. In comparison with conventional spirometry performed in primary care by very trained staff (technicians), the salary of hospital and primary care technicians in our public health system is similar, but in other systems, hospital technicians' salaries can be higher, which should be added to the total cost of the online procedure. Nevertheless, the additional cost does not seem too high, considering that the proposed procedure could solve the problem of performing technically acceptable spirometry in primary care.

In conclusion, spirometry performed online can be a good alternative to conventional spirometry. Its use could be reserved for intermediate and small primary care centres and hospitals without a pulmonary function laboratory.

\section{SUPPORT STATEMENT}

This study was supported by FIS (Fondo de Investigación Sanitaria) PI0040727, Ministerio de Ciencia e Innovación (SAF2008-02991) and SEAR (Sociedad Extremeña de Aparato Respiratorio) 2004.

\section{STATEMENT OF INTEREST}

None declared.

\section{ACKNOWLEDGEMENTS}

We are indebted to V. Rodríguez (Consejería de Educación, Junta de Extremadura, Cáceres, Spain) and V. Iglesias (Hospital San Pedro de Alcántara, Cáceres) for editing assistance in preparing the manuscript, and M. Caballé and I. Pérez (both Hospital San Pedro de Alcántara) for technical assistance.

\section{REFERENCES}

1 Sobradillo V, Miravitlles M, Gabriel R, et al. Geographical variations in prevalence and underdiagnosis of COPD in Spain.
Results of the IBERPOC multicenter epidemiological study. Chest 2000; 118: 981-989.

2 Murray CJ, Lopez AD. Alternative projections of mortality and disability by cause 1990-2020: Global Burden of Disease Study. Lancet 1997; 349: 1498-1504.

3 Bednarek M, Gorecka D, Wielgomas J, et al. Smokers with airway obstruction are more likely to quit smoking. Thorax 2006; 61: 869-873.

4 Tashkin DP, Celli B, Senn S, et al. A 4-year trial of tiotropium in chronic obstructive pulmonary disease. N Engl J Med 2008; 359: 1543-1554.

5 Bednarek M, Maciejewski J, Wozniak M, et al. Prevalence, severity and underdiagnosis of COPD in the primary care setting. Thorax 2008; 63: 402-407.

6 Levy ML, Fletcher M, Price DB, et al. International Primary Care Respiratory Group (IPCRG) Guidelines: diagnosis of respiratory diseases in primary care. Prim Care Respir J 2006; 15: 20-34.

7 General Practice Airways Group, International Primary Care Respiratory Group. Spirometry. www.theipcrg.org/resources/ spirometry.pdf Date last accessed: March 11, 2009. Date last updated: February 4, 2009.

8 Poels PJP, Schermer TRJ, Schellekens DPA, et al. Impact of a spirometry expert system on general practitioners' decision making. Eur Respir J 2008; 31: 84-92.

9 Miravitlles M, Llor C, de Castellar R, et al. Validation of the COPD severity score for use in primary care: the NEREA study. Eur Respir J 2009; 33: 519-527.

10 Calverley P. Fulfilling the promise of primary care spirometry. Eur Respir J 2008; 31: 8-10.

11 Caramori G, Bettoncelli G, Tosatto R, et al. Underuse of spirometry by general practitioners for the diagnosis of COPD in Italy. Monaldi Arch Chest Dis 2005; 63: 6-12.

12 Eaton T, Withy S, Garrett JE, et al. Spirometry in primary care practice. The importance of quality assurance and the impact of spirometry workshops. Chest 1999; 116: 416-423.

13 White $\mathrm{P}$, Wong $\mathrm{W}$, Fleming $\mathrm{T}$, et al. Primary care spirometry: test quality and the feasibility and usefulness of specialist reporting. Br J Gen Pract 2007; 57: 701-705.

14 Miller MR, Hankinson J, Brusasco V, et al. Standardisation of spirometry. Eur Respir J 2005; 26: 319-338.

15 Ferguson GT, Enright PL, Buist AS, et al. Office spirometry for lung health assessment in adults: a consensus statement from the National Lung Health Education Program. Respir Care 2000; 45: 513-530.

16 Pauwels RA, Buist AS, Calverley PM, et al. Global strategy for the diagnosis, management, and prevention of chronic obstructive pulmonary disease. NHLBI/WHO Global Initiative for Chronic Obstructive Lung Disease (GOLD) Workshop summary. Am J Respir Crit Care Med 2001; 163: 1256-1276.

17 Maree DM, Videler EA, Hallauer M, et al. Comparison of a new desktop spirometer (Diagnosa) with a laboratory spirometer. Respiration 2001; 68: 400-404.

18 Miravitlles M, Fernández I, Guerrero T, et al. Development and results of a screening program for COPD in primary care. The PADOC Project (Program for the Increase in the Diagnosis of COPD in Primary Care). Arch Bronconeumol 2000; 36: 500-505.

19 Liistro G, Vanwelde C, Vincken W, et al. Technical and functional assessment of 10 office spirometers: a multicenter comparative study. Chest 2006; 130: 657-665.

20 Lin K, Watkins B, Johnson T, et al. Preventive Services Task Force. Screening for chronic obstructive pulmonary disease using spirometry: summary of the evidence for the US Preventive Services Task Force. Ann Intern Med 2008; 148: 535-543.

21 Derom E, van Weel C, Liistro G, et al. Primary care spirometry. Eur Respir J 2008; 31: 197-203.

22 Schermer T, Eaton T, Pauwels R, et al. Spirometry in primary care: is it good enough to face demands like World COPD Day? Eur Respir J 2003; 22: 725-727. 
23 Hansen JE, Sun X-G, Wasserman K. Should forced expiratory volume in six seconds replace forced vital capacity to detect airway obstruction? Eur Respir J 2006; 27: 1244-1250.

24 Jing JY, Huang TC, Cui W, et al. Should FEV1/FEV6 replace FEV1/ FVC ratio to detect airway obstruction? A metaanalysis. Chest 2009; 135: 991-998.

25 Bellia V, Pistelli R, Catalano F, et al. Quality control of spirometry in the elderly. The SA.R.A. study. Salute Respiration nell'Anziano $=$ Respiratory Health in the Elderly. Am J Respir Crit Care Med 2000; 161: 1094-1100.

26 Schermer TR, Jacobs JE, Chavannes NH, et al. Validity of spirometric testing in a general practice population of patients with chronic obstructive pulmonary disease (COPD). Thorax 2003; 58: 861-866.

27 Finkelstein J, Cabrera MR, Hripcsak G. Internet-based home asthma telemonitoring: can patients handle the technology? Chest 2000; 117: 148-155.

28 Morlion B, Knoop C, Paiva M, et al. Internet-based home monitoring of pulmonary function after lung transplantation. Am J Respir Crit Care Med 2002; 165: 694-697.

29 Pellegrino R, Viegi G, Brusasco V, et al. Interpretative strategies for lung function tests. Eur Respir J 2005; 26: 948-968.

30 Lung function testing: selection of reference values and interpretative strategies. American Thoracic Society. Am Rev Respir Dis 1991; 144: 1202-1218.

31 Hyatt RE, Cowl CT, Bjoraker JA, et al. Conditions associated with an abnormal nonspecific pattern of pulmonary function tests. Chest 2009; 135: 419-424.
32 Quanjer PH, Tammeling GJ, Cotes JE, et al. Lung volumes and forced ventilatory flows. Report working party standardization of lung function test, European Community for Steel and Coal. Official statement of the European Respiratory Society. Eur Respir J 1993; 6: Suppl. 16, 5-40.

33 Dowson LJ, Mushtaq M, Watts T, et al. A re-audit of pulmonary function laboratories in the West Midlands. Respir Med 1998; 92: 1155-1162.

34 Menezes AM, Victora CG, Perez-Padilla R, et al. The Platino project: methodology of a multicenter prevalence survey of chronic obstructive pulmonary disease in major Latin American cities. BMC Med Res Methodol 2004; 4: 15.

35 Stoller JK, Buist AS, Burrows B, et al. Quality control of spirometry testing in the registry for patients with severe $\alpha 1$-antitrypsin deficiency. $\alpha_{1}$-Antitrypsin Deficiency Registry Study Group. Chest 1997; 111: 899-909.

36 Lum EH, Gross TJ. Telemedical education: teaching spirometry on the Internet. Am J Physiol 1999; 276: S55-S61.

37 American Thoracic Society, Medical Section of the American Lung Association. Standardization of spirometry. 1994 update. Am J Respir Crit Care Med 1995; 152: 1107-1136.

38 Burgos F, Disdier C, Roger N, et al. Tele-collaboration enhances quality of forced spirometry in primary care. Am J Respir Crit Care Med 2008; 77: A144.

39 Farre R, Navajas D. Quality control: a necessary, but sometimes overlooked, tool for improving respiratory medicine. Eur Respir J 2009; 33: 722-723. 